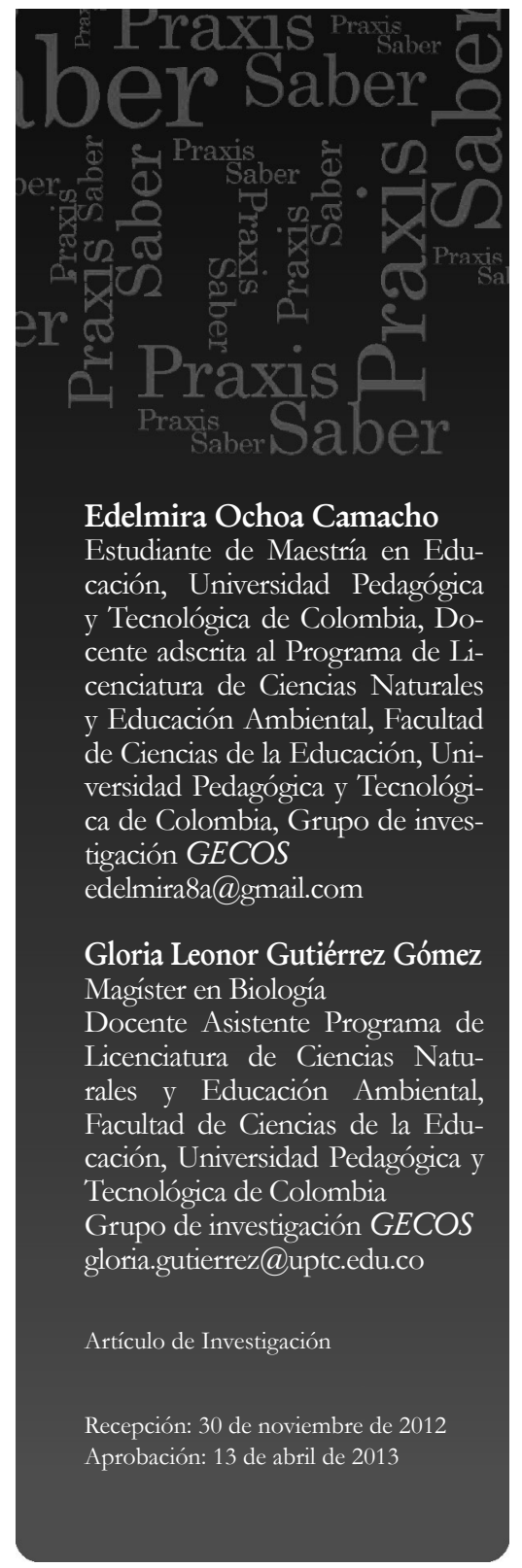

\title{
ACTITUDES FRENTE A LA RELACIÓN CTS DE ESTUDIANTES DE LICENCIATURA EN CIENCIAS NATURALES
}

\section{Resumen}

El trabajo de investigación que presenta este artículo se desarrolló en el marco de la tesis de Maestría en Educación de la Universidad Pedagógica y Tecnológica de Colombia entre los años 2010 y 2012. Uno de sus objetivos fue evaluar las actitudes de los estudiantes universitarios de primer semestre de la Licenciatura en Ciencias Naturales y Educación Ambiental frente a la relación Ciencia, Tecnología y Sociedad (CTS), y su diseño metodológico se enmarcó dentro de la investigación cuantitativa, con enfoque descriptivo, que permitió a la vez hacer análisis cualitativos, para conocer e identificar puntos fuertes y débiles de las actitudes hacia dichos temas. Para tal propósito los estudiantes diligenciaron el Cuestionario de Opiniones de Ciencia Tecnología y Sociedad (COCTS) y se realizó el análisis de la información.

Los resultados globales exhiben actitudes con valores cercanos a cero y el análisis detallado por cuestiones, categorías y frases permite identificar diversidad de creencias que configuran las actitudes previas de los estudiantes involucrados. Se concluyó que la utilización del COCTS y su 
análisis aporta a una mejor comprensión e identificación de las actitudes de los estudiantes encuestados sobre la relación CTS.

Palabras clave: actitud, ciencia, tecnología, estudiantes universitarios, alfabetización científica.

\title{
Natural Sciences University Students' Attitude to THE RELATION SCTS
}

\begin{abstract}
The research work presented in this paper was developed in the frame of the master thesis in Education of the Universidad Pedagógica y Tecnológica de Colombia between the years 2010 and 2012. One of its objectives was to assess Natural Science and Environmental Education first semester university students's attitude to the relation Science, Technology, and Society (ScTS). The methodological design was framed by the quantitative research with descriptive approach which helped to make qualitative analysis to know and identify strong and weak points of their attitudes to those topics. In order to do so, students filled in a Questionnaire about Opinions on Science, Technology and Society (QOScTS) and after that the data was analyzed. Global results show attitudes with values close to zero while the detailed analysis of questions, categories, and phrases enables to identify a wide variety of beliefs that shape participants' previous attitudes. It was concluded that the use of QOScTS and its analysis provide a better understanding and identification of the survey group's attitudes to the relation ScTS.
\end{abstract}

Key words: attitude, science, technology, university students, scientific literacy.

\section{Attitudes des Etudiants de Licence en Sciences Naturelles Face À la Relation StS}

\section{Résumé}

Le travail de recherche présenté dans cet article se développe dans le cadre du Mémoire de Master en Éducation de l'Université Pédagogique et Technologique de Colombie des années 2010 à 2012. Un de ses objectifs a été d'évaluer les attitudes des étudiants universitaires de premier semestre 
de Licence en Sciences Naturelles et Éducation Environnementale face à la relation Sciences, Technologie et Société (STS), et son design méthodologique s'est inscrit dans le cadre de la recherche quantitative, avec une approche descriptive, qui a permis à la fois de réaliser des analyses qualitatives pour connaitre et identifier les points forts et les points faibles des attitudes envers ces thèmes. À cet effet, les étudiants ont remplis le Questionnaire d'Opinions de Science Technologie et Société (QOSTS) et une analyse de l'information a été réalisée. Les résultats globaux démontrent des attitudes avec des valeurs proches de zéro et l'analyse détaillée par questions, catégories et phrases permet d'identifier une diversité de croyances qui configurent les attitudes préalables des étudiants impliqués. Il a été conclu que l'utilisation du QOSTS et son analyse ont apporté une meilleure compréhension et identification des attitudes des étudiants interrogés face à la relation STS.

Mots clés: attitude, sciences, technologie, étudiants universitaires, alphabétisation scientifique.

\section{Atitudes Frente À Relação Cts de Alunos de Licenciatura em CiÊnCias Naturais}

\section{Resumo}

O artigo apresenta resultados de um projeto de pesquisa de dissertação de mestrado em educação na Universidad Pedagógica y Tecnológica de Colombia nos anos 2010 a 2012. Um dos objetivos foi avaliar as atitudes dos alunos de graduação no primeiro semestre da Licenciatura em Ciências Naturais e Educação Ambiental, frente à relação Ciência, Tecnologia e Sociedade (CTS), e sua metodologia centrou-se no paradigma quantitativo, com enfoque descritivo que permitiu também fazer analises qualitativos para conhecer e identificar pontos fortes e fracos das atitudes na frente de ditos temas. Para esse propósito os alunos preencheram o questionário de opiniões de Ciência, Tecnologia e Sociedade (COCTS) e realizou-se a analise da informação. Os resultados globais exibem atitudes com valores perto de zero e a análise detalhada por questões, categorias e frases permite identificar diversidade de crenças que configuram as atitudes previas dos alunos envolvidos. Concluiu-se que a utilização do COCTS e sua análise aportam uma melhor compreensão e identificação das atitudes dos alunos da pesquisa sobre a relação CTS.

Palavras chave: atitude, ciência, tecnologia, alunos de faculdade, alfabetização cientifica. 


\section{Introducción}

Entre los años 2010 y 2012 se desarrolló, como requisito para optar al título de Magíster en Educación en la Universidad Pedagógica y Tecnológica de Colombia (UPTC), el trabajo de investigación que se presenta a continuación. Fue realizado en el marco del Proyecto Iberoamericano de Evaluación de Actitudes Relacionadas con la Ciencia, la Tecnología y la Sociedad (PIEARCTS); estudio de investigación cooperativa internacional en el que participan diversos grupos de investigación pertenecientes a distintos países, instituciones y regiones de lenguas ibéricas (español y portugués). La perspectiva del estudio es fundamentalmente educativa y se centra en los temas acerca de cómo funcionan la ciencia y la tecnología en el mundo actual, la naturaleza de la ciencia y la tecnología y las relaciones entre la Ciencia, la Tecnología y la Sociedad (CTS) que son un componente esencial en la alfabetización científica.

En el contexto colombiano son pocas las investigaciones realizadas al respecto, y particularmente en la ciudad de Tunja no existen estudios que evalúen y caractericen las actitudes frente a la relación CTS de estudiantes universitarios de Licenciatura de Ciencias Naturales y Educación Ambiental, lo cual nos motivó a participar en este estudio cooperativo. Así, hemos decidido centrar nuestra mirada en las actitudes frente a los temas CTS de los futuros licenciados (estudiantes de primer semestre) del programa de Ciencias Naturales con el fin de indagar sobre el estado de sus actitudes y analizar la contribución real de los currículos (educación básica y media), utilizando una nueva metodología que permite tanto el análisis cualitativo como la aplicación cuantitativa de la estadística inferencial para la investigación de estos temas, lo cual ofrece validez y fiabilidad (Manassero et ál., 2001).

El objetivo general de este trabajo de investigación fue evaluar las actitudes de los estudiantes universitarios de primer, octavo y décimo semestre de la Licenciatura en Ciencias Naturales y Educación Ambiental, frente a la relación CTS. En este artículo se presentan los resultados correspondientes a uno de los tres grupos participantes en el estudio, en este caso, estudiantes de primer semestre, y como objetivos específicos, se identificaron las actitudes, debilidades y fortalezas frente a la relación CTS de los estudiantes universitarios de primer semestre, utilizando el cuestionario COCTS forma 1, infiriéndose algunos posibles factores que pueden influir en las actitudes de la población objeto de estudio para así 
proponer algunas recomendaciones. En este sentido, un análisis de las actitudes de los docentes en formación pone en evidencia elementos para asumir este reto.

La inclusión de las relaciones CTS en la enseñanza de las ciencias puede contribuir no sólo a mejorar la actitud y a aumentar el interés hacia la ciencia y su aprendizaje sino también puede favorecer aprender más ciencia y saber más sobre ciencia, al mostrar una imagen más completa y socialmente contextualizada de la misma (Vilches \& Furió, 1999).

El estudio de las actitudes CTS permitirá cuestionar el hecho de que "los currículos de ciencias suelen centrarse sobre todo en los contenidos conceptuales y regirse por la lógica interna de la ciencia, pero se olvidan de dar formación sobre la ciencia misma, es decir, sobre qué es la ciencia, cómo funciona internamente, cómo se desarrolla, cómo construye su conocimiento, cómo se relaciona con la sociedad, qué valores utilizan los cientificos en su trabajo profesional, etc.” (Acevedo et ál., 2007).

Para la educación científica, la evaluación inicial de las actitudes de los profesores en formación sobre los temas CTS es útil ya que puede contribuir a la mejora de éstas, máxime si se tiene en cuenta la idea, evidente en sí misma, de que solamente se puede enseñar aquello que se entiende, de modo que si se desea desarrollar en los futuros licenciados una comprensión más adecuada sobre la relación CTS, es necesario examinar las debilidades y fortalezas de formación para alcanzar este objetivo.

De otra parte, varias investigaciones relacionadas con estudios sobre actitudes CTS, muestran de manera reiterada que profesores y estudiantes no alcanzan una comprensión adecuada sobre los temas CTS, indispensable para tener una población informada sobre aspectos científico-tecnológicos necesarios para la participación democrática y la toma de decisiones (Vázquez et ál., 2005). En otros estudios se expresa que uno de los objetivos explícitos en el proceso de educar debería ser fomentar actitudes positivas hacia la ciencia y el trabajo científico para preparar personas que respondan a las exigencias de la vida, y este objetivo es especialmente relevante en la formación de los futuros profesores (Gil-Pérez \& Vilches, 2001). Por todo lo anterior, creemos que los resultados de este estudio contribuirán a un mejor conocimiento y comprensión de las actitudes de los profesores en formación y a analizar la influencia que tienen los currículos para la mejora de la comprensión de los temas CTS de la población participante. 
Del mismo modo, consideramos en sintonía con el proyecto PIEARCTS que un mayor conocimiento y comprensión de la problemática que se investiga puede contribuir a la articulación de propuestas de mejora de la educación científica sobre los temas CTS, en particular si de formación docente se trata, y en sí aportar elementos interesantes para el fortalecimiento de la didáctica de la enseñanza de las ciencias. También nos interesa que los resultados obtenidos sean una aportación para generar espacios de reflexión en el programa para la mejora de la comprensión de la naturaleza de la ciencia en la formación de licenciados en ciencias, así como el fortalecimiento del rol docente para la nueva sociedad del conocimiento.

$\mathrm{Al}$ respecto, es pertinente indicar que para el desarrollo del proyecto el término actitud se usó para referirse a las ideas sobre los temas CTS por considerarlo más válido teóricamente (Manassero et ál., 2004), pues permitió describir el tipo de aprendizajes que implican dichos temas al integrar simultáneamente componentes cognitivos, afectivos y conductuales (Manassero et ál., 2001). En el lenguaje habitual el término actitud tiene ciertas connotaciones de estado de ánimo, incluso morales e ideológicas, pero en el marco de la psicología social (marco desde el cual se emplea el término en el proyecto), sintetizando centenares de contribuciones, la actitud es un concepto que reúne tres elementos: Un conjunto organizado y duradero de convicciones o creencias (elemento cognitivo), dotadas de una predisposición o carga afectiva favorable o desfavorable (elemento evaluativo o afectivo), que guían la conducta de la persona respecto a un determinado objeto social (elemento conductual).

Así, la disposición a favor o en contra del objeto de la actitud (elemento afectivo o evaluativo) es considerada por muchos autores lo más característico y propio de ésta, lo que la sitúa en el ámbito de los valores, la dota de capacidad para orientar la conducta de las personas y sugiere las connotaciones ideológicas; es decir, hace de las actitudes un constructo con connotaciones de motivación guía de la conducta de las personas. El dinamismo y amplitud del concepto actitud le conecta con otros términos (hábitos, creencias, valores, opiniones, etc.) hasta el punto que suele ser habitual una tendencia reduccionista a identificarlas con ellos.

\section{Metodología}

Este trabajo investigativo se enmarcó en una investigación de base cuantitativa de tipo descriptivo que también permitió realizar análisis cualitativos e identificar puntos débiles y fuertes de las actitudes de los 
estudiantes encuestados frente a la relación CTS (Lederman et ál., 2002 citado en Vázquez et ál., 2010). Los puntos fuertes son las actitudes que tienen los estudiantes que son coincidentes con los conocimientos de los expertos provenientes de la historia, la sociología y la filosofía de la ciencia y la tecnología actuales, lo que significa que en el caso de una frase categorizada como adecuada los estudiantes afirman su acuerdo hacia esta frase lo mismo que los expertos; en el caso de las frases categorizadas como ingenuas los estudiantes niegan su acuerdo con la frase lo mismo que la niegan los expertos y para el caso de las frases categorizadas como plausibles los estudiantes afirman su carácter ambivalente. Los puntos débiles se refieren a las actitudes opuestas a los conocimientos de los expertos que tienen los estudiantes de los temas CTS; tal oposición, significa en el caso de frases categorizadas como adecuadas, que los estudiantes están en desacuerdo con esa frase, negando lo que afirman los expertos; en el caso de las frases categorizadas como ingenuas, significa que los estudiantes están de acuerdo con la frase, afirmando lo que niegan los expertos, y en el caso de las frases plausibles se oponen a su carácter ambivalente afirmando su acuerdo o desacuerdo con éstas (Vázquez et ál., 2010).

Según Vázquez et ál., "la normalización de los indices ofrece precisión cuantitativa al diagnóstico, asociando los indices más positivos a puntos fuertes y los indices más negativos a los puntos débiles. El análisis del contenido de las frases con los indices más positivos y más negativos profundiza cualitativamente en las actitudes más fuertes y más débiles de los estudiantes” (2010). Para el ámbito educativo, la identificación de los puntos fuertes y débiles de las actitudes frente a la relación CTS de los docentes en formación de Licenciatura en Ciencias Naturales y Educación Ambiental es relevante, ya que puede ser utilizada como guía para conocer e identificar las actitudes, y un punto de partida para iniciar procesos de reflexión sobre la propia práctica que permita a corto y mediano plazo plantear y proponer procesos de intervención didáctica y curricular como mecanismos necesarios de mejora basados en la investigación.

\section{Muestra}

Los participantes en este estudio son cuarenta y un estudiantes de primer semestre de Licenciatura de Ciencias Naturales y Educación Ambiental. La edad aproximada de la muestra se extiende entre 16 y 22 años con un promedio aproximado de 19 años y está configurada por un $66 \%$ de mujeres y $34 \%$ de hombres. 


\section{Instrumento}

El Cuestionario de Opiniones sobre Ciencia, Tecnología y Sociedad (COCTS), está acreditado como uno de los mejores instrumentos de papel y lápiz para evaluar las actitudes sobre los temas y la relación CTS referidos como naturaleza de la ciencia, una creación reciente del grupo de investigadores del proyecto PIEARCTS (Vázquez et ál., 2005). El COCTS es un conjunto de cien cuestiones de opción múltiple independientes entre sí, y que pueden usarse de forma flexible y abierta. Este instrumento ha sido sometido a análisis crítico por parte de especialistas y jueces para mejorar el diseño de algunas cuestiones y categorías del escalamiento de las frases de las cuestiones a lo largo de varias etapas (Vásquez et ál., 2005), por lo cual es considerado por los expertos como un instrumento válido y fiable para el estudio de las actitudes sobre la relación CTS.

Cada cuestión del COCTS plantea un problema en el encabezado sobre el que se desea conocer la actitud de los estudiantes participantes en el estudio, seguido de una lista de frases alternativas (posibles respuestas) al problema planteado y que están ordenadas y etiquetadas sucesivamente con una letra (A, B, C, D, E, etc.). El grado de acuerdo personal frente a cada posible respuesta es expresado a través del uso de una escala de 1 a 9 o de ser necesario a partir del uso de una de dos expresiones: "No entiendo" (E) o "No sé" (S).

Las quince cuestiones aplicadas en el estudio, que contienen noventa y nueve frases opcionales ubicadas en cuarenta y tres categorías (adecuadas, plausibles e ingenuas), pertenecen a cinco dimensiones del COCTS (Tabla 1).

Tabla 1. Selección consensuada de la ubicación en la taxonomía (dimensiones) de las quince cuestiones del COCTS aplicadas en la investigación.

\begin{tabular}{|c|c|}
\hline Dimensiones & F1 - 15 Cuestiones \\
\hline \multirow{2}{*}{ a) Definición de Іа СуТ } & F1_10111 Ciencia \\
\hline & F1_10411 Interdependencia \\
\hline \multirow{4}{*}{$\begin{array}{l}\text { b) Sociología externa de la ciencia } \\
\text { Influencia triádica } \\
\text { Influencia de la sociedad en CyT }\end{array}$} & \\
\hline & F1_30111 Interacción CTS \\
\hline & F1_20141 Política del gobierno de un país \\
\hline & F1_20411 Ética \\
\hline \multirow[t]{3}{*}{ Influencia de Ia СуТ en la sociedad } & F1_40161 Responsabilidad social/contaminación \\
\hline & F1_40221 Decisiones morales \\
\hline & F1_40531 Bienestar social \\
\hline \multirow{4}{*}{ c) Sociología interna de Ia C yT } & F1_60111 Motivaciones \\
\hline & F1_60611 Infrarrepresentación de mujeres \\
\hline & F1_70231 Decisiones por consenso \\
\hline & F1_80131 Ventajas para la sociedad \\
\hline \multirow[t]{3}{*}{ d) Epistemología } & F1_90211 Modelos científicos \\
\hline & F1_90411 Provisionalidad \\
\hline & F1_90621 Método científico \\
\hline
\end{tabular}




\section{Procedimiento}

Los estudiantes encuestados responden al problema planteado en cada cuestión, según un Modelo de Respuesta Múltiple (Tabla 2) donde cada estudiante valora, sobre una escala de nueve puntos (Tipo Likert) el grado de acuerdo (1) o desacuerdo (9) con todas y cada una de las opciones presentes en la cuestión. El formato de opción múltiple permite a los encuestados expresar sus puntos de vista sobre todas las frases que configuran cada cuestión, de tal manera que la actitud expresada mediante la valoración de las diferentes frases que configuran las cuestiones es más rica, precisa y completa.

Tabla 2. Modelo de respuesta múltiple para una cuestión del COCTS. Significado de las puntuaciones directas de acuerdo/desacuerdo con cada frase alternativa, asignaciones de puntos en la escala de valoración y los procedimientos de cálculo de los índices actitudinales desde las puntuaciones directas.

\begin{tabular}{|c|c|c|c|c|c|c|c|c|c|c|c|c|c|c|c|c|}
\hline \multirow{2}{*}{\begin{tabular}{|r|} 
Categorías \\
Escala \\
\end{tabular}} & \multirow{2}{*}{$\begin{array}{l}\text { Número } \\
\text { de } \\
\text { posiciones } \\
\text { irecta }\end{array}$} & \multicolumn{9}{|c|}{$\begin{array}{c}\text { Escala de valoración: } \\
\text { transformación de las puntuaciones directas }\end{array}$} & \multicolumn{3}{|c|}{$\begin{array}{c}\text { Puntuaciones } \\
\text { actitudinales directas }\end{array}$} & \multicolumn{3}{|c|}{$\begin{array}{l}\text { Índice de actitud } \\
\text { de categoría }\end{array}$} \\
\hline & & 9 & 8 & 7 & 6 & 5 & 4 & 3 & 2 & 1 & Máx & Fórmula & Min & Máx & Fórmula & Min \\
\hline \multicolumn{2}{|c|}{ Grado de acuerdo } & Total & $\begin{array}{l}\text { Casi } \\
\text { total }\end{array}$ & Alto & $\begin{array}{c}\text { Parcial } \\
\text { alto }\end{array}$ & Parcial & $\begin{array}{c}\text { Parcial } \\
\text { Bajo }\end{array}$ & Bajo & $\begin{array}{l}\text { Casi } \\
\text { nulo }\end{array}$ & Nulo & & & & & & \\
\hline Adecuadas & $N_{a}$ & 4 & 3 & 2 & 1 & 0 & -1 & -2 & -3 & -4 & $+4 N$ & $\sum a_{j}$ & $-4 N_{a}$ & +1 & $I_{*}=\sum a_{j} / 4 N_{*}$ & -1 \\
\hline \begin{tabular}{|l} 
Plausibles \\
\end{tabular} & $N_{p}$ & -2 & -1 & 0 & 1 & 2 & 1 & 0 & -1 & -2 & $+2 \mathrm{~N}$ & $\sum p_{j}$ & $-2 N_{p}$ & +1 & $I_{p}=\sum p_{j} / 2 N_{p}$ & -1 \\
\hline Ingenuas & $N_{n}$ & -4 & -3 & -2 & -1 & 0 & 1 & 2 & 3 & 4 & $+4 N$ & $\sum n_{j}$ & $-4 N_{n}$ & +1 & $I_{n}=\sum n_{j} / 4 N_{n}$ & -1 \\
\hline Total & $\mathrm{N}$ & & & & & & & & & & Índice & de actitud & global & 1 & $I=\left(I_{0}+I_{g}+I_{n}\right) / 3$ & -1 \\
\hline
\end{tabular}

Fuente: Manassero et ál. (2004)

$a_{j}$ : puntuación de variación directa para las frases "adecuada" $\mathrm{j}$

$p_{j}$ : puntuación de variación directa para las frases "plausible" j $n_{j}$ : puntuación de variación directa para las frases "ingenua" $\mathrm{j}$

$\sum$. suma de las puntuaciones directas desde $j=1$ a $j=N_{a}\left(j=N_{p} o j=N_{n}\right)$ para el conjunto de las frases pertenecientes a cada una de las categorías "adecuada" "plausible" "ingenua"

Las puntuaciones directas se transforman en un índice actitudinal normalizado en el intervalo $(-1,+1)$ para luego interpretarlo como medias actitudinales, teniendo en cuenta la categoría de cada frase: adecuada que expresa una opinión apropiada desde la perspectiva de los conocimientos de la historia, la filosofía y la sociología de la ciencia (puntuación 7, 8 o 9), plausible, que aunque no es completamente adecuada, expresa algunos aspectos apropiados desde la perspectiva de los conocimientos de la 
historia, la filosofía y la sociología de la ciencia (puntuación 4, 5 o 6), e ingenua que expresa una opinión inapropiada o no plausible (puntuación 1, 2 o 3), asignadas previamente por un panel de jueces. Para obtener los índices actitudinales normalizados $(-1,+1)$ para todas las frases se divide la valoración resultante de la escala por el rango, en función de la categoría asignada a cada una de las frases (aj /4, pj /2, nj/4 respectivamente). En esta escala de valoración, cuanto más positivo y cercano al valor máximo $(+1)$ es un índice, la actitud es considerada más adecuada e informada y cuanto más negativo (-1) y cercano a la unidad es un índice, representa una actitud más ingenua o desinformada (Manassero et ál., 2001).

En la Tabla 2 se señalan los valores asignados para las puntuaciones directas: así, los índices actitudinales de cada frase se transforman en índices de las tres categorías (adecuadas, plausibles e ingenuas) promediando el valor del índice de las frases que pertenecen a cada una de las tres categorías existentes en la cuestión. A su vez, el promedio de los índices de las tres categorías de cada cuestión produce un índice global de cada cuestión, que es el indicador de la actitud global hacia el tema planteado en cada cuestión (Vázquez et ál., 2010).

El criterio general para delimitar las frases con las actitudes más positivas o más negativas y determinar las diferencias más relevantes entre grupos, se basa en aplicar una puntuación de corte de (0.30 unidades) a las puntuaciones medias y al tamaño del efecto de las diferencias; por debajo de este umbral las puntuaciones o las diferencias se consideran irrelevantes (Benássar et ál., 2011).

\section{Resultados}

Para realizar una primera aproximación a la descripción estadística los parámetros que se tuvieron en cuenta fueron el índice actitudinal máximo y mínimo, la media y la desviación estándar de cada frase, categoría y cuestión para la muestra. Como resultado se obtuvieron las grandes medias globales de los promedios de los índices de cada una de las cuestiones, categorías y frases que se interpretan como representativas para la muestra objeto de estudio.

\section{Resultados estadísticos descriptivos por cuestiones}

La Tabla 3 muestra los indicadores más globales de las posiciones de los estudiantes de primer semestre sobre los temas CTS planteados en las 
quince cuestiones sometidas a su consideración. En conjunto el análisis de la distribución de las medias de cada una de las cuestiones indica un sesgo leve hacia valores actitudinales ligeramente positivos para toda la muestra, pues la gran media de las puntuaciones medias de las cuestiones es positiva pero próxima a cero $(+0,0562$ puntos D.E $=0,2439)$. Esto es, se obtiene un valor global para las cuestiones muy bajo de las actitudes CTS de los estudiantes de primer semestre. Las actitudes más positivas aparecen en los temas de responsabilidad social por la contaminación e infrarrepresentación de las mujeres. Las cuestiones con los índices más negativos son: influencia de la cultura ética o religiosa en la ciencia y la tecnología y motivaciones de los científicos respecto a la ciencia y la tecnología. Sólo uno de estos valores $(>0,3211)$ apenas supera el valor del umbral establecido como indicador relevante.

Tabla 3. Estadísticos descriptivos básicos de los índices ponderados en cada una de las quince cuestiones.

\begin{tabular}{|l|l|r|r|r|}
\hline \multirow{2}{*}{\multicolumn{2}{c||}{ Cuestiones }} & \multicolumn{3}{c|}{ Estudiantes primer semestre } \\
\cline { 3 - 5 } & N & \multicolumn{1}{c|}{ Media } & \multicolumn{1}{c|}{ D.E } \\
\hline \hline F1_10111 & Ciencia & 41 & 0,0820 & 0,1658 \\
\hline F1_10411 & Interdependencia & 41 & 0,1902 & 0,2577 \\
\hline F1_20141 & Política del gobierno de un país & 41 & 0,1823 & 0,2070 \\
\hline F1_20411 & Ética & 41 & $-0,1463$ & 0,2887 \\
\hline F1_30111 & Interacción CTS & 41 & 0,1141 & 0,3078 \\
\hline F1_40161 & Responsabilidad social contaminación & 41 & 0,3211 & 0,2930 \\
\hline F1_40221 & Decisiones sociales & 41 & $-0,0071$ & 0,2372 \\
\hline F1_40531 & Bienestar mejor nivel de vida & 41 & 0,1108 & 0,2395 \\
\hline F1_60111 & Motivaciones & 41 & $-0,1425$ & 0,2183 \\
\hline F1_60611 & Infrarrepresentación de las mujeres & 41 & 0,2020 & 0,2562 \\
\hline F1_70231 & Decisiones por consenso & 41 & $-0,1159$ & 0,2254 \\
\hline F1_80131 & Ventajas para la sociedad & 41 & 0,1122 & 0,2296 \\
\hline F1_90211 & Modelos científicos & 41 & $-0,0287$ & 0,2553 \\
\hline F1_90411 & Provisionalidad & 41 & $-0,0107$ & 0,2156 \\
\hline F1_90621 & Método científico & 41 & $-0,0207$ & 0,2608 \\
\hline
\end{tabular}

\section{Resultados estadísticos descriptivos por categorías}

Siguiendo el proceso sugerido por los expertos del proyecto PIEARCTS, los índices de cada una de las tres categorías (adecuadas, ingenuas y plausibles) de cada cuestión se obtienen como promedio de los índices de las frases que forman cada categoría. Los índices de las categorías, al referirse a un menor número de frases, reflejan una mayor variabilidad que los índices de las cuestiones globales, en los cuales se engloban varias 
frases de la misma categoría, exhibiendo valores más altos tanto positivos como negativos (Vázquez et ál., 2010).

En las tablas se muestran sólo los índices actitudinales positivos y negativos seleccionados teniendo en cuenta el punto de corte $(>0,30$ puntos) o (<-0,30 puntos) (recomendado por Benássar et ál., 2011).

\section{* Actitudes de los estudiantes de primer semestre por categorías}

La gran media de todos los índices de las categorías sigue siendo positiva pero baja $(\mathrm{m}=+0,076$ puntos; D.E $=0,412)$ indicando que la actitud global de los estudiantes, medida a través de los índices de las categorías, es poco satisfactoria.

En la Tabla 4 se muestran los índices actitudinales por categorías (adecuado AD, plausible PL e ingenuo IN) seleccionados tanto positivos como negativos. Así, de cuarenta y tres categorías que configuran la Forma 1 del COCTS, catorce categorías presentan valores relevantes tanto positivos como negativos, de las cuales hay ocho categorías adecuadas, cinco categorías ingenuas y sólo una categoría plausible. Las categorías con los índices más positivos son la mayoría de las categorías adecuadas y en menor medida las categorías ingenuas (dos categorías) de cada cuestión. Tres de las cinco categorías ingenuas obtienen valores negativos y la categoría plausible que exhibe el valor más negativo del conjunto de categorías de la Tabla 4.

Los valores más altos en los índices de las categorías adecuadas (AD) se obtienen en los temas: responsabilidad social por la contaminación, bienestar social e interdependencia. Los valores con los índices más negativos en las categorías se obtienen en los temas relacionados con las motivaciones (plausible PL) y en las categorías ingenuas (IN) en los temas: ventajas para la sociedad, ética y decisiones por consenso. 
Tabla 4. Estadísticos descriptivos básicos de los índices de las categorías con las puntuaciones medias más altas positivas y más bajas negativas de los estudiantes de primer semestre.

\begin{tabular}{|c|c|c|c|}
\hline Categorías & $\mathrm{N}$ & Media & D.E \\
\hline Índice adecuadas responsabilidad social/contaminación F1_40161AD & 41 & 0,6179 & 0,3646 \\
\hline Índice adecuadas bienestar social F1_40531AD & 41 & 0,5793 & 0,3768 \\
\hline Índice adecuadas Interdependencia F1_10411AD & 41 & 0,5183 & 0,3828 \\
\hline Índice adecuadas método científico F1_90621AD & 41 & 0,439 & 0,4375 \\
\hline Índice adecuadas decisiones por consenso F1_70231AD & 41 & 0,4268 & 0,4303 \\
\hline Índice adecuadas interacción CTS F1_30111AD & 41 & 0,3933 & 0,3628 \\
\hline Índice adecuadas provisionalidad F1_90411AD & 41 & 0,378 & 0,4074 \\
\hline Índice ingenuas responsabilidad social/contaminación F1_40161IN & 41 & 0,3537 & 0,6892 \\
\hline Índice ingenuas infrarrepresentación de las mujeres F1_60611IN & 41 & 0,3394 & 0,416 \\
\hline Índice adecuadas ventajas para la sociedad F1_80131AD & 41 & 0,3252 & 0,346 \\
\hline Índice ingenuas decisiones por consenso F1_70213IN & 41 & $-0,3171$ & 0,2992 \\
\hline Índice ingenuas ética F1_20411IN & 41 & $-0,3293$ & 0,5425 \\
\hline Índice ingenuas ventajas para la sociedad F1_80131IN & 41 & $-0,4146$ & 0,3456 \\
\hline Índice plausibles motivaciones F1_60111PL & 41 & $-0,5244$ & 0,591 \\
\hline
\end{tabular}

\section{Resultados descriptivos estadísticos por frases}

Los índices actitudinales de las frases individuales del COCTS expresan las actitudes directas de los estudiantes de primer semestre sobre la afirmación específica propuesta en cada frase. La especificidad del contenido de cada frase favorece que estos índices actitudinales presenten mayores variaciones en sus puntuaciones que las categorías o las cuestiones completas. De otra parte, el gran número de frases analizadas del COCTS, también contribuye a que haya mayor número de frases con índices actitudinales que superen el valor de punto de corte considerado como relevante $(>0,30$ o $<-0,30$, sugerido por Benássar et ál., 2011) por lo que a continuación se presentan por separado las frases con las puntuaciones medias más altas (positivas) y las frases con las puntuaciones medias más bajas (negativas), para el grupo de estudiantes participantes.

\section{* Actitudes de los estudiantes de primer semestre por frases}

Los índices actitudinales de las frases individuales del cuestionario que tienen las puntuaciones más altas y positivas por encima del punto de corte son veintiséis, equivalentes al 26,3\% del total de las frases 
del cuestionario. La mayoría de las frases con índices muy positivos pertenecen a las categorías de frases adecuadas, aunque también hay algunas frases ingenuas (6); en este grupo de índices muy positivos, también es destacable la ausencia de frases de la categoría plausible (Tabla 5).

Las frases con los índices más positivos corresponden a doce de las quince cuestiones que conforman el cuestionario, de las cuales hay dos cuestiones que presentan cuatro frases cada una (40161, 60611), dos cuestiones que presentan tres frases cada una (20141, 30111), cuatro cuestiones que presentan dos frases cada una (10111, 10411, 40531, 80131) y cuatro cuestiones que presentan una frase cada una (70231, 90211, 90411, 40221). Las tres frases con los índices más positivos corresponden a tres cuestiones (40161 F, $40531 \mathrm{E}, 10411 \mathrm{~B})$. La primera cuestión se refiere a la responsabilidad social por la contaminación, la segunda al bienestar social, y la tercera a la interdependencia de la ciencia y la tecnología. Las frases con el índice actitudinal más positivo son las siguientes:

a) La industria pesada no debería trasladarse a los países no desarrollados porque la contaminación debería ser limitada tanto como sea posible. Extenderla solo crearía más daños.

b) Más tecnología mejorará el nivel de vida de nuestro país. Sí y no. Más tecnología haría la vida más agradable y más eficiente, pero también causaría más contaminación, desempleo y otros problemas. El nivel de vida puede mejorar pero la calidad de vida puede que no.

C) La ciencia y la tecnología están estrechamente relacionadas entre sí porque la investigación científica conduce a aplicaciones prácticas tecnológicas, y las aplicaciones tecnológicas aumentan la capacidad para hacer investigación científica.

Es importante destacar que de la totalidad de las cuestiones aplicadas (15) en el cuestionario completo presentan alguna de sus frases situadas en el grupo de frases más positivas, es decir que en todos los temas planteados hay alguna actitud de los estudiantes que resulta muy positiva; la única excepción es la cuestión 90211, referida a modelos científicos, que no tiene ninguna frase entre las más positivas. Para la educación científica y la formación inicial de docentes, conocer las actitudes tanto positivas como negativas frente a los temas CTS contribuirá para estructurar estrategias didácticas que fortalezcan y enriquezcan las actitudes positivas con las 
que llegan los estudiantes a la universidad, así como para mejorar aquellas que son ingenuas o ambiguas.

Otro rasgo destacable es que las veintiséis frases con los índices más positivos fueron evaluadas por algunos estudiantes participantes igual que las evaluaron los expertos (estas frases se identifican con la etiqueta _C_), es decir que también se pueden identificar actitudes adecuadas de los estudiantes participantes que corresponden con actitudes acerca de la relación CTS consensuadas en la comunidad científica (Tabla 5).

Tabla 5. Estadísticos descriptivos básicos de los índices con las puntuaciones medias más altas positivas en las frases opcionales (la letra entre guiones indica el carácter adecuado _A_o ingenuo _I_ de cada frase; "las frases con _C_ representan actitudes consensuadas por los expertos, identificadas por algunos estudiantes participantes como adecuadas").

\begin{tabular}{|c|c|c|c|c|}
\hline \multicolumn{2}{|r|}{ Frases } & \multirow{2}{*}{\begin{tabular}{l|l}
$N$ \\
41
\end{tabular}} & \multirow{2}{*}{\begin{tabular}{|r|} 
Media \\
0,671
\end{tabular}} & \multirow{2}{*}{$\begin{array}{l}\text { D. E } \\
0,424\end{array}$} \\
\hline F1_C_A_40161F & Responsabilidad social/contaminación & & & \\
\hline F1_C_A_40531E & Bienestar social & 41 & 0,671 & 0,442 \\
\hline F1_C_A__10411B & Interdependencia & 41 & 0,628 & 0,404 \\
\hline F1_C_A_40161C & Responsabilidad social/contaminación & 41 & 0,598 & 0,496 \\
\hline F1_C_A_40161D & Responsabilidad social/contaminación & 41 & 0,585 & 0,566 \\
\hline F1_C_A_40221B & Decisiones morales & 41 & 0,567 & 0,296 \\
\hline F1_C_I_10111I & Ciencia & 41 & 0,543 & 0,545 \\
\hline F1_C_A_10111B & Ciencia & 41 & 0,531 & 0,437 \\
\hline F1_C_I_60611A & Infrarrepresentación de las mujeres & 41 & 0,518 & 0,563 \\
\hline F1_C_A_40531D & Bienestar social & 41 & 0,488 & 0,429 \\
\hline F1_C_A_20141F & Gobierno política de un país & 41 & 0,488 & 0,379 \\
\hline F1_C_I_20141J & Gobierno política de un país & 41 & 0,445 & 0,511 \\
\hline F1_C_A_90621C & Método Científico & 41 & 0,439 & 0,414 \\
\hline F1_C_I_30111G & Interacción CTS & 41 & 0,433 & 0,578 \\
\hline F1_C_A_70231C & Decisiones por consenso & 41 & 0,427 & 0,430 \\
\hline F1_C_A_10411C & Interdependencia & 41 & 0,409 & 0,496 \\
\hline F1_C_A_60611F & Infrarrepresentación de las mujeres & 41 & 0,409 & 0,398 \\
\hline F1_C_I_60611B & Infrarrepresentación de las mujeres & 41 & 0,396 & 0,524 \\
\hline F1_C_A_30111E & Interacción CTS & 41 & 0,396 & 0,451 \\
\hline F1_C_A_30111F & Interacción CTS & 41 & 0,390 & 0,600 \\
\hline F1_C_A_90411B & Provisionalidad & 41 & 0,378 & 0,407 \\
\hline F1_C_A__80131B & Ventajas para la sociedad & 41 & 0,372 & 0,455 \\
\hline F1_C_A_60111H & Motivaciones & 41 & 0,354 & 0,589 \\
\hline F1_C_I_40161A & Responsabilidad social/contaminación & 41 & 0,354 & 0,689 \\
\hline F1_C_A_80131E & Ventajas para la sociedad & 41 & 0,342 & 0,417 \\
\hline F1_C_A_20141B & Gobierno política de un país & 41 & 0,317 & 0,494 \\
\hline
\end{tabular}


El análisis cualitativo del contenido de las frases con los índices más positivos revela los puntos fuertes de las actitudes de los estudiantes acerca de la relación CTS, es decir, las actitudes de los estudiantes que coinciden con los conocimientos de los expertos desde la historia, la sociología y la filosofía de CyT (Vázquez et ál., 2010).

Los índices actitudinales de las frases individuales del cuestionario que tienen las puntuaciones más bajas y negativas (Tabla 6), con puntuaciones por debajo del punto de corte, son nueve frases (y en ellas están representadas siete cuestiones de las cuales siete frases pertenecen a la categoría ingenua y las otras dos frases a la categoría plausible. En este grupo de índices negativos se destaca la ausencia de las frases de la categoría adecuada (Tabla 6). Entre las frases más negativas se destacan las frases de la categoría ingenua (70231A) que se refiere a la toma de decisiones por consenso en la comunidad científica, la frase $60111 \mathrm{G}$ que se refiere a motivaciones y la frase de la categoría plausible infrarrepresentación de las mujeres $(60611 \mathrm{H})$. Asimismo, siete de estas frases negativas fueron evaluadas por algunos estudiantes participantes como ideas ingenuas o plausibles coincidentes con la evaluación realizada por los expertos (estas frases se identifican con la etiqueta _C_) (Tabla 6). Las frases con la etiqueta _C_ corresponden a actitudes consensuadas por los expertos, es decir actitudes sobre las cuales no existe controversia pues se admiten por los especialistas como actitudes ingenuas o plausibles acerca de la ciencia y la tecnología (Vázquez et ál., 2010).

Tabla 6. Estadísticos descriptivos básicos de los índices con las puntuaciones medias más bajas negativas en las frases opcionales (la letra entre guiones indica el carácter ingenuo _I_o plausible_P_de cada frase; "las frases con _C_ representan actitudes consensuadas por los expertos e identificadas por los estudiantes de primer semestre como adecuadas").

\begin{tabular}{|c|c|c|c|c|}
\hline \multicolumn{2}{|r|}{ Frases } & $\mathrm{N}$ & Media & D. E \\
\hline F1_C_I_90411C & Provisionalidad & 41 & $-0,311$ & 0,394 \\
\hline F1_C_I_70231F & Decisiones por consenso & 41 & $-0,317$ & 0,542 \\
\hline F1_I_90211C & Modelos científicos & 41 & $-0,329$ & 0,508 \\
\hline F1_C_I_20411G & Ética & 41 & $-0,390$ & 0,642 \\
\hline F1_C_I_80131A & Ventajas para la sociedad & 41 & $-0,415$ & 0,456 \\
\hline F1_C_P_60111E & Motivaciones & 41 & $-0,476$ & 0,536 \\
\hline F1_C_P_60611H & Infrarrepresentación de mujeres & 41 & $-0,524$ & 0,591 \\
\hline F1_C_I_60111G & Motivaciones & 41 & $-0,549$ & 0,494 \\
\hline F1_I_70231A & Decisiones por consenso & 41 & $-0,604$ & 0,362 \\
\hline
\end{tabular}


Las frases con el índice actitudinal más negativo son respectivamente las siguientes:

a) Los científicos que proponen una teoría deben convencer a otros científicos mostrándoles pruebas concluyentes que apoyen que la teoría es verdad.

b) La mayoría de los científicos están motivados para esforzarse mucho en su trabajo. La razón principal de su motivación personal para hacer ciencia es: descubrir nuevas ideas o inventar cosas para beneficio de la sociedad (por ejemplo remedios médicos, soluciones a la contaminación, etc.)

c) No existen razones para tener más científicos que científicas. Ambos son igualmente capaces de ser buenos en ciencia y hoy en día las oportunidades son similares.

\section{Conclusiones}

De acuerdo con los antecedentes y los aspectos de los temas CTS considerados para el estudio de las actitudes de los estudiantes se puede inferir que existe un nivel bajo en el conocimiento y comprensión de la relación CTS.

Los resultados globales muestran una tendencia de las actitudes de los estudiantes encuestados fundamentadas en diversidad de conceptos tanto positivos como negativos que estructuran las actitudes frente a la relación CTS (ver Tabla 3). Así, han mostrado algunas actitudes específicas más adecuadas, pero en la mayoría de casos combinadas con otras ingenuas, empobreciendo la actitud global. La actitud global muestra que el estudiantado tiene deficiencias importantes en el conocimiento y comprensión de la mayoría de los aspectos de la naturaleza de la ciencia o CTS del contenido científico que aprende. De un modo más específico se puede concluir que los estudiantes tienen una actitud ingenua sobre la mayoría de los aspectos que configuran la naturaleza de la ciencia o temas CTS.

Las actitudes negativas y poco informadas de los estudiantes sobre los temas CTS corresponden mayoritariamente a frases categorizadas como ingenuas y en menor medida plausibles, mientras que las actitudes positivas e informadas corresponden mayoritariamente a frases categorizadas como adecuadas y en menor medida de la categoría 
ingenua, con ausencia de frases de la categoría plausible (ver Tablas 5 y 6). Varias de estas actitudes tanto positivas como negativas coinciden con actitudes relatadas en la literatura (Lederman., 2002; Manassero et ál., 2001; Vázquez et ál., 2010). Aunque existen actitudes más informadas, se observan actitudes ingenuas, tradicionales y hasta contradictorias, que indican deficiencias en el conocimiento y comprensión de los temas CTS, y que expresan debilidad en la contribución de los currículos con respecto a la formación científica (ver Tablas 4,5 y 6).

Este resultado reclama una enseñanza de las ciencias que tenga en cuenta los objetivos del enfoque CTS, que favorezca el desarrollo de actitudes en los estudiantes más adecuadas respecto a los valores de la ciencia y la tecnología, y que a su vez contribuya para la orientación de una ciencia más asequible a todas las personas, necesaria para alcanzar la alfabetización científica y tecnológica (Manassero et ál., 2001).

Históricamente el proceso enseñanza aprendizaje de las ciencias ha estado dominado por una visión tradicional que ha ponderado determinadas formas de enseñanza por sobre el aprendizaje significativo, que no es eficaz para promover cambios conceptuales y que no tiene en cuenta los aspectos actuales de los temas CTS, necesarios para promover la alfabetización científica de los estudiantes. En el aula durante la práctica se muestra también una ausencia de los factores asociados a cómo se produce el conocimiento, los valores y la ética de la ciencia, la influencia que tiene la ciencia en la sociedad y la cultura, así como ausencia de la ciencia y la tecnología en la vida cotidiana de los estudiantes, que la mayor parte de las veces impiden la comprensión sobre el contenido científico tecnológico que se enseña.

Para la educación científica y la formación continua de docentes es relevante tener en cuenta las actitudes frente a la relación CTS con las cuales ingresan los estudiantes al programa, para ser reforzadas o para ser mejoradas a través de los semestres con el fin de evaluar el proceso enseñanza aprendizaje así como la contribución que está haciendo el currículo del programa para la mejora de estas actitudes y el fortalecimiento del rol docente.

Teniendo en cuenta los resultados obtenidos por el grupo y dado que las relaciones CTS son débiles, se sugiere que dentro de la cualificación que reciben los docentes del programa de Licenciatura se revisen estos 
resultados obtenidos y al respecto se involucren actividades, talleres, conferencias, entre otros, que traten el enfoque CTS dado que éste ya está incluido dentro de los estándares básicos de ciencias naturales, ya que los futuros licenciados en Ciencias Naturales y Educación Ambiental deben conocerlo y aplicarlo en su quehacer docente.

Finalmente, se evidencia que la utilización del COCTS y el análisis multivarial aporta a una mejor comprensión de la complejidad de las actitudes de los estudiantes encuestados sobre los temas CTS (Lederman et ál., 2002), pues se matizan aspectos de las actitudes que resultan relevantes en los procesos de identificación de actitudes sobre la relación CTS, en la formación docente y en la necesaria reorientación para una mejor enseñanza de las ciencias.

\section{Referencias}

Acevedo, J. A.; Vázquez, A.; Manassero Mas, M. A. \& Acevedo, R. (2007). 'Consensos sobre la naturaleza de la ciencia: Fundamentos de una investigación empírica'. Revista Eureka Divulgación científica. Recuperado de http://redalyc.uaemex.mx/pdf/920/92040104.pdf

Benássar, R. A; Vázquez, A; Manassero Mas, M.A \& García-Carmona, A. (2011). Ciencia, Tecnología y Sociedad en Iberoamérica: Una evaluación de la comprensión de la naturaleza de la ciencia y la tecnología. Recuperado el 28 de noviembre de 2011 de http://www. oei.es/salactsi/DOCUMENTO5vf.pdf

Callejas, M. \& Mendoza, E. (2011). 'Diferencias en la comprensión de la $\mathrm{NdCy} \mathrm{T}$ entre profesores en formación que inician la universidad y los que finalizan su grado'. Ciencia, Tecnología y Sociedad en Iberoamérica: Una evaluación de la comprensión de la naturaleza de la ciencia y la tecnología.

Giordan, A. \& Sanmartino, M. (s.f.). 'Educación Científica y Tecnológica'. Revista Novedades Educativas. Buenos Aires: México. Recuperado de http://www.ldes.unige.ch/info/membres/sanmartino/articles/ esp/GiordanSanmartino2.pdf

GIL-PÉrez, D. \& Vilches, A. (2001). Una alfabetización científica para el siglo XXI. Obstáculos y propuestas de actuación. Recuperado de http://www.oei.es/catmexico/una_alfabetizacion_cientifica\%20.pdf

Lederman, N. G.; Abd - Khalick, F.; Bell, R. L \& Schwartz (2002). 'Views of nature of science questionnaire (VNOS): Toward valid 
and meaningful assessment of learners' conceptions of nature of science'. Journal of Research in Science Teaching, 39, 6, (pp. 497 - 521). Recuperado de http://www.gb.nrao.edu/ sheather/For_ Sarah $/ 1$ it $\% 20$ on $\% 20$ nature $\% 20$ of $\% 20$ science/views $\% 20$ of $\% 20$ nature $\% 20$ of $\% 20$ science $\% 20$ questionnaire.pdf

Manassero, M. A.; Vázquez, A \& Acevedo, J.A. (2001). 'La evaluación de las actitudes CTS'. Sala de lectura CTS +I. Recuperado de http:// www.oei.es/salactsi/acevedo11.htm

(2004). 'Evaluación de las actitudes del profesorado respecto a los temas CTS: Nuevos avances metodológicos'. Revista electrónica Enseñanza de las ciencias.

Recuperado de http://www.raco.cat/index.php/ensenanza/article/ viewFile/21980/21814

VÁzQuez, A.; Castillejos, A.; García-Ruíz, M.; Garritz, A.; Manassero, M.; Martín, M.; Quetglas, B. \& Rueda, C. (2005). Proyecto Iberoamericano de Actitudes Relacionadas con la Ciencia, la Tecnología y la Sociedad (PIEARCTS).

VÁzquez, A.; Manassero, M. A \& Talavera, M. (2010). 'Actitudes y creencias sobre la naturaleza de la ciencia y la tecnología en una muestra representativa de jóvenes estudiantes'. Revista electrónica de enseñanza de las ciencias, Vol. 9, N²2, (pp. 333-335). Recuperado de http://www.saum.uvigo.es/reec/volumenes/volumen9/ART3_ Vol9_N2.pdf

Vilches, A. \& Furió, C. (1999). 'Ciencia Tecnología Sociedad: Implicaciones de la Educación Científica para el siglo XXI'. Sala de Lectura CTS. Recuperado de http://www.oei.es/salactsi/ctseducacion.htm 